\title{
DNA Damage, Repair, and Diseases
}

\author{
Lisa Wiesmüller, ${ }^{1}$ James M. Ford, ${ }^{2}$ and Robert H. Schiest ${ }^{3}$ \\ ${ }^{1}$ University of Hamburg, Germany \\ ${ }^{2}$ Stanford University School of Medicine, California, USA \\ ${ }^{3}$ UCLA Schools of Medicine and Public Health, California, USA
}

DNA is the essential carrier of genetic information in all living cells. How is the huge amount of DNA in organisms from bacteria to humans maintained and protected from the ravages of noxious agents in the environment? The chemical stability of the DNA molecule is not unusually great, DNA undergoes several types of spontaneous modifications, and it can also react with many physical and chemical agents, of which some are endogenous products of the cellular metabolism (eg, reactive oxygen species) while others, including ionizing radiation and ultraviolet light, are threats from the external environment. The resulting alterations of DNA structure are generally incompatible with its essential role in preservation and transmission of genetic information. Damage to DNA can cause genetic alterations, and if genes that control cell growth are involved, these mutations can lead to the development of cancer. Of course, DNA damage may also result in cell death which can have serious consequences for the organism of which the cell is a part; for example, loss of irreplaceable neurons in the brain. Accumulation of damaged DNA has also been considered to contribute to some of the features of aging. It is not surprising that a complex set of cellular surveillance and repair mechanisms has evolved to reverse the potentially deleterious damage that would otherwise destroy the precious blueprint for life. Some of these DNA repair systems are so important that life cannot be sustained without them. An increasing number of human hereditary diseases that are characterized by severe developmental problems and/or a predisposition to cancer have been found to be linked to deficiencies in DNA repair.

The many types of DNA repair include excision repair mechanisms targeted to the removal of bulky DNA adducts and UV-induced photoproducts, base-pair alterations and purine loss, and DNA mismatches, and single- and doublestrand DNA breaks. In addition, DNA replication, recombination and transcription are all involved in DNA repair pathways. A complex interplay between intrinsic hereditary factors and persisting DNA damage determines the susceptibility of humans to cancer. Inherited human diseases of DNA repair include many cancer susceptibility syndromes, such as Xeroderma pigmentosum, Ataxia-telangectasia, Bloom's and Werner's syndromes, Hereditary Non-Polyposis Colon Cancer, Li-Fraumeni-syndrome, and breast/ovarian cancer syndrome.

The most ubiquitous and versatile modes of DNA repair are those in which the damaged or incorrect part of a DNA strand is excised and then the resulting gap is filled by repair replication using the complementary strand as template. The excision repair pathway that deals with UV-irradiation induced pyrimidine dimers and a large variety of cancercausing chemical adducts to DNA is known as nucleotide excision repair (NER). Several very interesting new developments in this field will be reviewed. These include DNA damage inducible responses in both prokaryotic and eukaryotic organisms, including both inducible DNA repair genes and genes involved in the proteasomal/ubiquitination pathway and the detection of such inducible responses using wholegenome transcriptional profiling techniques.

Recombination represents the irreplaceable repair mechanism under circumstances when DSBs appear as a result of metabolic processes or genotoxic treatment. In mammalian cells about half of all DSBs are repaired by homologous recombination, half by nonhomologous end joining. The lack of central enzyme functions can cause lethal phenotypes of knockout mice or result in extreme sensitivities towards ionizing radiation. Moreover, correct repair of DSBs is central to the maintenance of genomic integrity in mammalian cells, since errors give rise to translocations, deletions, duplications, expansions, and transposon integration, which accelerate the multistep process of tumor progression. Therefore, recombination processes are subject to surveillance by a hierarchy of genome stabilizing factors, such as p53 and the PI3 kinase ATM.

Lisa Wiesmüller James M. Ford Robert H. Schiestl 

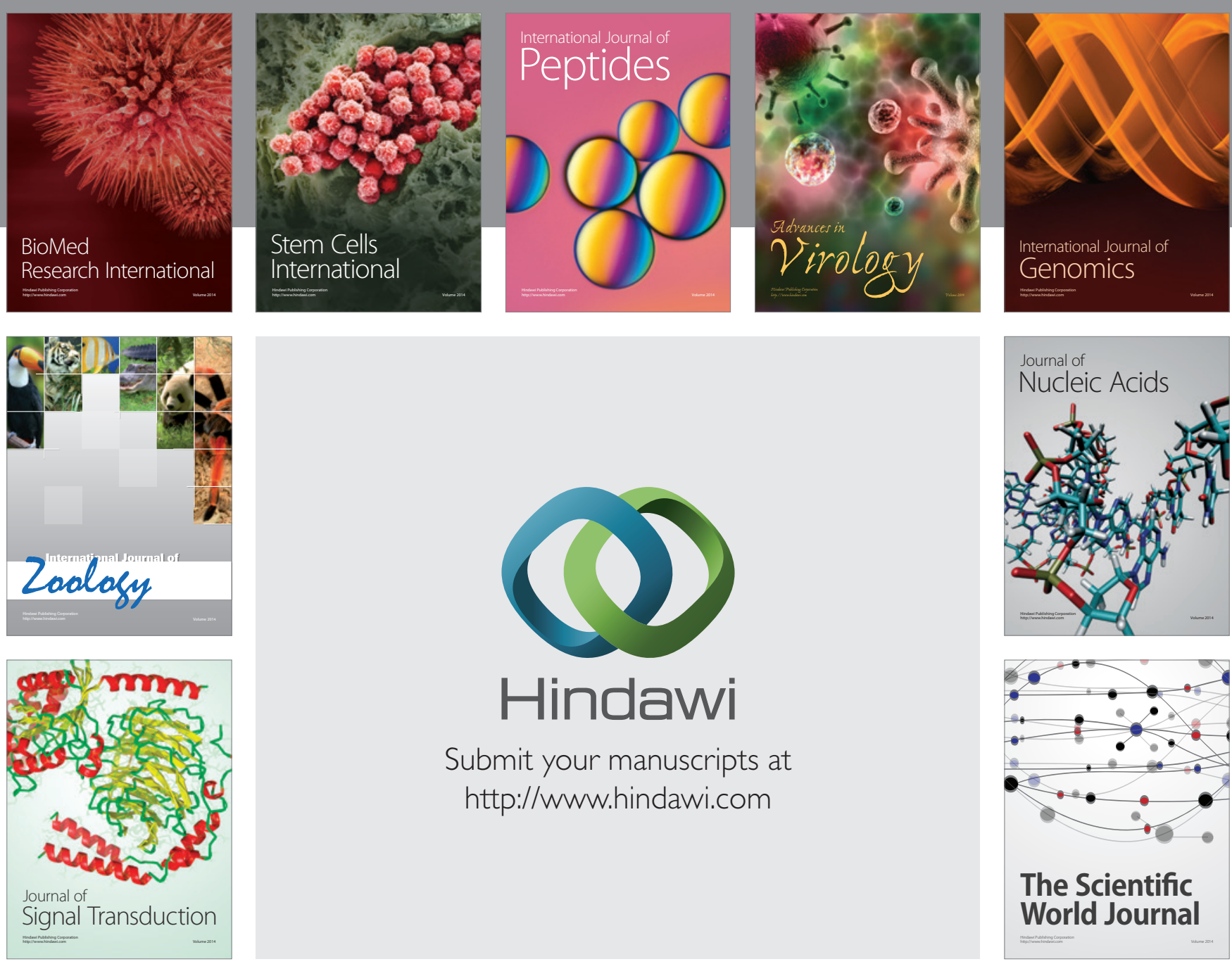

Submit your manuscripts at

http://www.hindawi.com
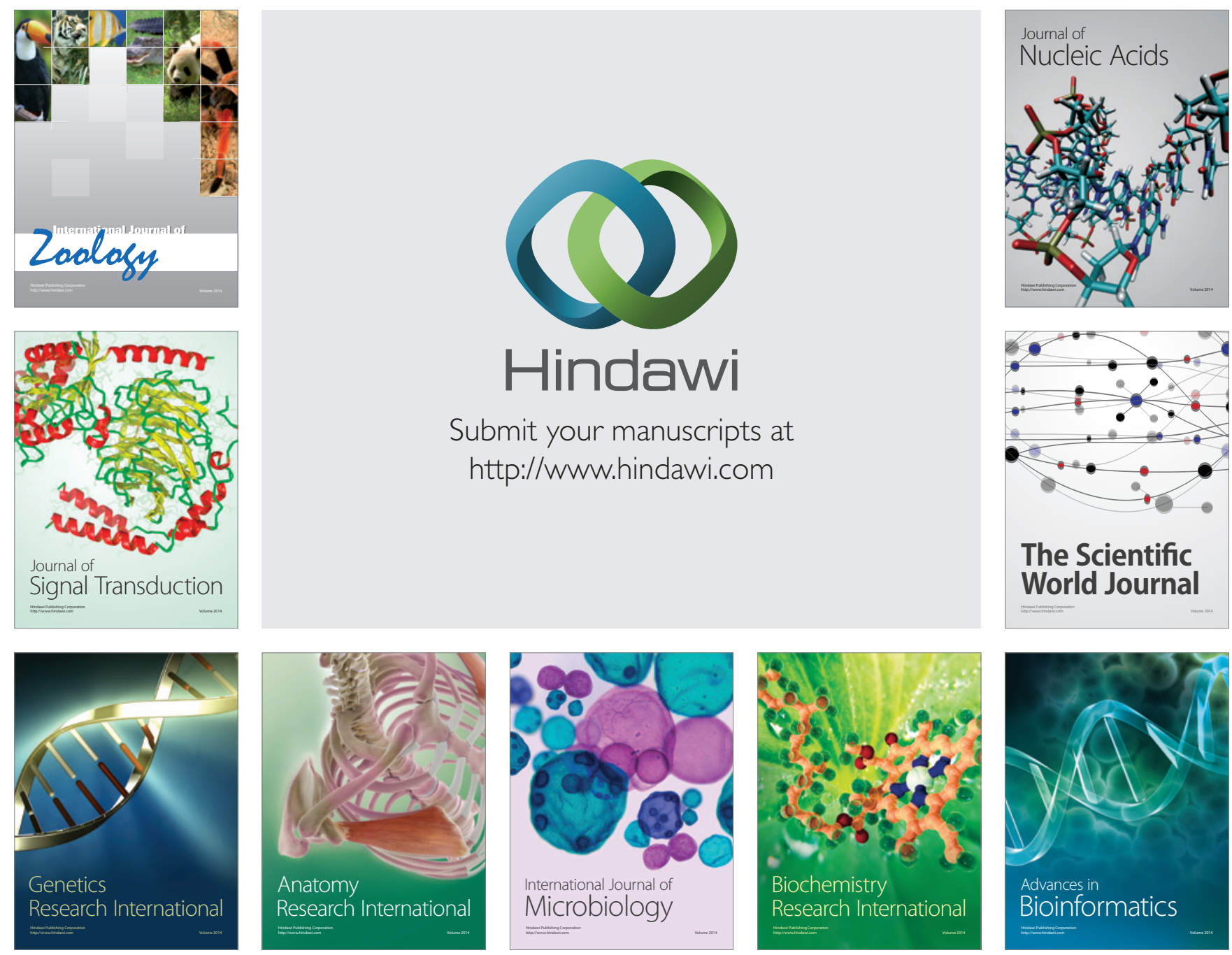

The Scientific World Journal
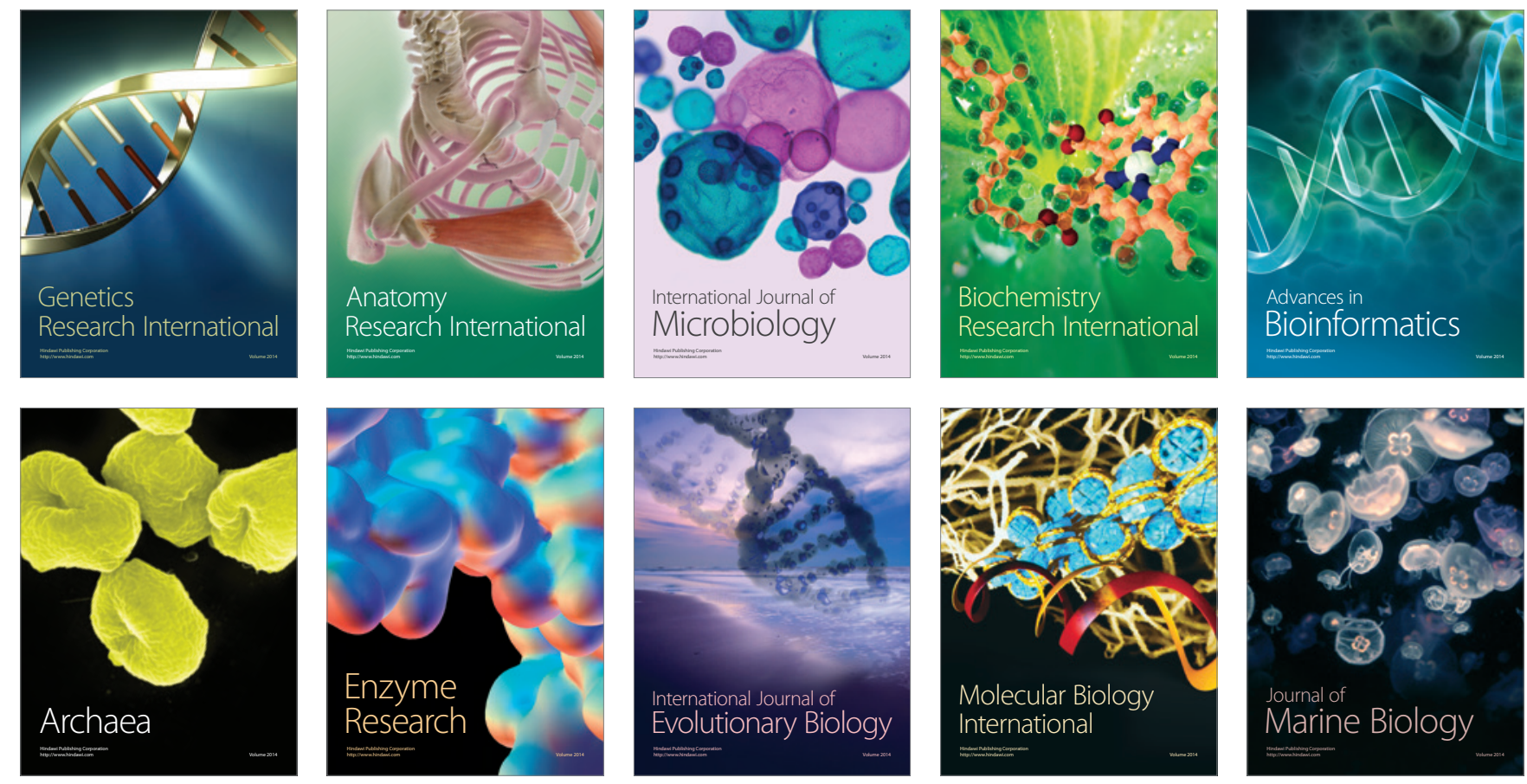